\title{
Recommandations pour les registres: rendre les données utiles
}

\author{
Christoph Bosshard \\ Dr méd., vice-président de la FMH, responsable du département Données, démographie et qualité (DDQ)
}

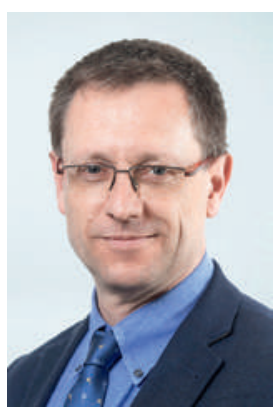

«C'est désespérant», me disent régulièrement des collègues à propos de la bureaucratisation galopante qui impacte aussi leurs patients. «Et voilà que tu nous amènes encore d'autres tâches administratives», poursuivent-ils lorsque je leur propose une nouvelle idée que je juge prometteuse concernant les registres.

Je réalise alors où sont les limites: pour convaincre les médecins et les patients, le travail supplémentaire doit non seulement être réduit au strict minimum, mais également leur apporter une plus-value claire. Ensuite, il faut instaurer un lien de confiance et leur assurer que le traitement des données collectées sera uniquement celui convenu et que toutes les précautions requises

Les données collectées doivent s'avérer utiles. En même temps, elles doivent être interprétées et utilisées avec précaution.

seront prises pour leur interprétation et leur utilisation. Nous savons tous qu'une découverte a ses limites, et qu'il est tout aussi important d'en connaître les limites que le bien-fondé. Prenons l'exemple de la surconsommation de prestations médicales où, comme dans tant d'autres domaines de la recherche, les conclusions ne sont possibles qu'à titre rétrospectif. C'est une réalité scientifique qui va à l'inverse de notre manière de vivre plutôt tournée vers l'avenir. Il est vrai que savoir d'où nous venons nous aide à fixer le cap pour l'avenir, mais cela ne nous exempte pas de devoir parcourir le chemin, toujours éclairés par notre expérience, notre savoir-faire médical, tout en pesant soigneusement nos décisions avec nos patients.

Le 29 août 2012, la FMH a publié un document de base qui a contribué à lancer la réflexion sur le thème des registres. S'agissant des différents standards possibles, un groupe de travail regroupant cinq organisations concernées, à savoir l'ANQ, H+, l'ASSM, unimedsuisse et la FMH, a vu le jour dans le but d'établir un vaste consensus. Les recommandations concernant la création et la gestion de registres médicaux ont été adoptées après que le plus grand nombre d'intervenants possible a été consulté et les quelque 160 retours reçus intégrés.
Cette démarche "de bas en haut» (bottom-up) dans le but de recueillir l'avis du terrain doit montrer au monde politique et à l'administration ce qu'il est nécessaire de faire pour arriver à des conclusions fiables, sans oublier que, là aussi, la question des ressources revêt une importance centrale.

Pour que la qualité des registres puisse être vérifiée de manière efficace, les auteurs des recommandations ont établi des standards minimaux visant notamment à garantir la qualité des données, mais aussi leur saisie et leur utilisation selon des critères clairement définis. S'agissant de la gestion des registres, la question de la compétence a également été abordée. Les recommandations ont aussi pour objectif de garantir en tout temps le respect du droit de la personnalité de ceux qui fournissent leurs données. Enfin, l'application de standards minimaux permet un relevé efficace des données et augmente leur utilité. Il est ainsi possible d'éviter les saisies à double, de mieux exploiter les synergies entre les lieux de collecte et d'améliorer la qualité et la portée des données.

Les différentes activités menées par les médecins à titre individuel ou collectif (organisations médicales) témoignent des nombreux efforts déjà entrepris dans ce domaine. Pour une vue d'ensemble des démarches ac-

Pour que la qualité des registres de santé puisse être vérifiée, des standards minimaux sont recommandés.

tuelles, la FMH met à disposition une plateforme des registres médicaux à l'adresse www.asqm.ch $\rightarrow$ Thèmes $\rightarrow$ Transfert de connaissances. A l'image de ce que la FMH et ses membres ont toujours fait et continueront de faire au quotidien, la FMH veut poser une pierre supplémentaire à l'édifice déjà très riche de la qualité en médecine, grâce aux recommandations communes de H+, l'ANQ, l'ASSM, unimedsuisse et la FMH publiées dans le Bulletin des médecins suisses du 12 octobre 2016. Vous les trouverez également sur www.asqm.ch $\rightarrow \mathrm{Pu}-$ blications $\rightarrow$ Recommandations registres. 